\title{
Mood and Implicit Alcohol Expectancy Processes: Predicting Alcohol Consumption in the Laboratory
}

\author{
Jeffrey D. Wardell, M.A., \\ Department of Psychology, The State University of New York at Buffalo \\ Jennifer P. Read, Ph.D., \\ Department of Psychology, The State University of New York at Buffalo \\ John J. Curtin, Ph.D., and \\ Department of Psychology, University of Wisconsin—Madison, Madison, WI \\ Jennifer E. Merrill, M.A. \\ Department of Psychology, The State University of New York at Buffalo
}

\begin{abstract}
Background-Implicit positive alcohol expectancy (PAEs) processes are thought to respond phasically to external and internal stimuli - including mood states - and so they may exert powerful proximal influences over drinking behavior. Although social learning theory contends that mood states activate mood-congruent implicit PAEs, which in turn lead to alcohol use, there is a dearth of experimental research examining this mediation model relative to observable drinking. Moreover, an expectancy theory perspective might suggest that, rather than influencing PAEs directly, mood may moderate the association between PAEs and drinking. To test these models, the present study examined the role of mood in the association between implicitly measured PAE processes (i.e., latency to endorse PAEs) and immediate alcohol consumption in the laboratory. Gender differences in these processes also were examined.
\end{abstract}

Method-College students ( $\mathrm{N}=146)$ were exposed to either a positive, negative, or neutral mood induction procedure, completed a computerized PAE reaction time (RT) task, and subsequently consumed alcohol ad libitum.

Results-The mood manipulation had no direct effects on drinking in the lab, making the mediation hypothesis irrelevant. Instead, gender and mood condition moderated the association between RT to endorse PAEs and drinking in the lab. For males, RT to tension reduction PAEs was a stronger predictor of volume of beer consumed and peak BAC in the context of general arousal (i.e., positive and negative mood) relative to neutral mood. RT to PAEs did not predict drinking in the lab for females.

Conclusions-The results show that PAE processes are important determinants of immediate drinking behavior in men, suggesting that biased attention to mood-relevant PAEs - as indicated by longer RTs - predicts greater alcohol consumption in the appropriate mood context. The findings also highlight the need to consider gender differences in PAE processes. This study underscores the need for interventions that target automatic cognitive processes related to alcohol use.

Correspondence concerning this article should be addressed to Jeffrey D. Wardell, Department of Psychology, 206 Park Hall, The State University of New York at Buffalo, Buffalo, NY 14260. Voice: (716) 645-0252. Fax: (716) 645-3801. jwardell@ buffalo.edu. 


\section{Keywords}

alcohol expectancies; implicit cognition; mood; gender

Positive alcohol expectancies (PAEs) - beliefs about the desirable effects of alcohol use are robust, proximal predictors of drinking (Goldman et al., 1999; Sher et al., 1996) and thus may be an important focal point in the prevention of problem drinking. Recently, there has been a growing interest in automatic or implicit PAE processes, which may be primarily stimulus driven and may have an immediate influence over drinking behavior (Tiffany, 1990; Wiers et al., 2006; Wiers and Stacy, 2006). However, few attempts have been made to study the link between PAEs and observable drinking behavior in the laboratory. Both social learning theory (SLT) and expectancy theory posit that alcohol expectancies have a mechanistic role in drinking, proximally influencing in-the-moment drinking behavior (see Maisto et al., 1999; Goldman et al., 1999). However, empirical tests of hypotheses derived from these theoretical models are limited.

\section{Multidimensionality of PAEs: Importance of Implicit Cognitions}

PAEs are beliefs about the positive effects of alcohol that are the result of learning and are represented in memory (Goldman et al., 1999). Many different types of PAEs have been identified (e.g., social, enhancement, tension reduction; Kushner et al., 1994). PAEs have been studied using self-report methods that are thought to tap into explicit (conscious, deliberate) cognitive processes, as well as behavioral (e.g., reaction time) methods that are thought to reflect implicit (unconscious, automatic) cognitive processes (see De Houwer, 2006; Wiers and Stacy, 2006; Wiers et al., 2002). The concept of implicit expectancy has been described in different ways. Here, we use the term implicit to refer to expectancy processes that are measured using indirect methods that do not rely on explicit self-report and are thought to reflect automatic cognitive processes (De Houwer, 2006).

Both explicitly and implicitly measured PAEs account for unique variance in self-reported drinking (Houben and Wiers, 2008; McCarthy and Thompsen, 2006; Palfai and Wood, 2001; Thrush and Wiers, 2007). Implicit PAE processes may be especially relevant as immediate determinants of drinking because of their automatic, stimulus-driven nature (Wiers and Stacy, 2006). Implicit PAE activation is also associated with craving and increased consumption, especially when self-control resources are depleted (Ostafin et al., 2008). Thus, implicit PAEs may be important targets for interventions. Currently, interventions tend to challenge only explicit beliefs about alcohol, leaving implicit PAEs intact (e.g., Wiers et al., 2005; see also Wiers and Stacy, 2006). Thus, the examination of implicit PAE processes as proximal determinants of actual drinking behavior has potential to inform the development of interventions that target implicit PAEs. Though some studies have examined alcohol consumption after priming PAEs (e.g., Roehrich and Goldman, 1995; Stein et al., 2000), there have been only a few that have implicitly measured individual differences in PAEs just prior to alcohol consumption (Ostafin et al., 2008; Palfai et al. 2000; Payne et al., 2008).

\section{Mood and Implicit PAE Processes}

Implicit alcohol expectancy processes are thought to be dynamic; that is, they become activated in response to contextual or situational cues (Krank and Wall, 2006). Mood state is one such cue (see Greeley and Oei, 1999; Simons et al., 2005), with both positive and negative moods serving as antecedents of drinking (Birch et al., 2008; Cooper et al., 1995). Moreover, the content of alcohol expectancies can be mapped onto two dimensions of affective space, arousal (i.e., aroused vs. sedated mood) and valence (i.e., pleasant vs. 
unpleasant mood; Goldman et al., 1999; Rather et al., 1992; Read et al., 2009). Mood may have an influence over implicit PAE processes in particular, as these processes are thought to respond automatically to phasic changes in external and internal stimuli, including mood (Krank and Wall, 2006; Wiers and Stacy, 2006). ${ }^{1}$

PAEs as a mediator of the mood-drinking association-According to social learning theory (SLT), PAE processes act as the mediating cognitive mechanism linking mood and alcohol use (Goldman et al., 1999; Maisto et al., 1999), such that mood sates selectively activate mood-relevant PAEs, which in turn lead to alcohol consumption (Birch et al., 2008; Friedman et al., 2009; Goldstein et al., 2004; Hufford, 2001). Positive moods may be associated specifically with expectations that alcohol can enhance positive mood, whereas negative moods may be associated specifically with expectations that alcohol can relieve negative mood.

Although some work supports this conceptualization (e.g., Birch et al., 2008; Read and Curtin, 2007), findings are far from uniform. For example, there are inconsistencies among studies with respect to which mood states affect PAEs and how individual differences influence these processes (e.g., Birch et al., 2004; Birch et al., 2008; Stewart et al., 2002). Also, virtually no experimental tests of the full mediated model exist, as little research has examined whether mood-induced activation of PAEs predicts subsequent observable drinking.

Another issue is that previous work in this area has tended to focus only on positive and negative mood valence and has overlooked the other dimension, arousal (Russell, 1980). Indeed, both positive and negative moods can involve heightened arousal, which may be very relevant for understanding mood-related PAE processes, given that alcohol has direct effects on arousal (both stimulating and depressing effects; Pohorecky, 1977). Moreover, many PAE items contain arousal content (e.g., tension reduction and activity enhancement PAEs, Kushner et al, 1994; see also Kramer and Goldman, 2003).

Mood as a moderator of the PAE-drinking association-Mediation of mood and drinking by PAEs has been a predominant conceptualization, but it is not the only one possible. One alternative, informed by alcohol expectancy theory, follows from the notion that there are pre-existing individual differences in the strength of implicit PAEs due to a number of stable individual difference factors (see Goldman et al., 1999; Mann et al., 1987). The degree to which these individual differences in implicit PAEs influence drinking behavior, though, may depend on whether they are relevant to the particular context. That is, mood states may moderate the implicit PAEs-drinking association, such that mood-relevant PAEs influence drinking only when the relevant mood state is experienced. This is consistent with the expectancy theory view that "the influence of the expectancy remains latent until circumstances are encountered that make the information relevant" (Stein et al., 2000, p. 107; see also Bolles, 1972). So, whereas SLT focuses on the "activation" of implicit PAEs by mood, which may then lead to drinking (mediation hypothesis), this alternative model suggests that pre-existing differences in implicit PAE strength may be more or less relevant for predicting drinking depending on mood context (moderation hypothesis). As there are so few data on the effects of these mood and PAE processes on immediate, observable drinking, we sought to test these associations in terms of both the mediation and moderation perspectives.

\footnotetext{
${ }^{1}$ We acknowledge that some studies have reported mood influences on explicitly measured PAEs (e.g., Birch et al., 2004; Grant and Stewart, 2007). However, these studies tend to measure explicit PAEs with instruments keyed to be more sensitive to dynamic changes in PAEs (e.g., "right now, alcohol would..."). We are not arguing that mood has no influence at all over explicit self-report of PAEs, only that mood states may have a greater impact on implicitly measured PAE processes such as latency to endorse PAEs.
} 
In order to examine how state-like, mood-related PAE processes influence an immediate drinking episode, it is important to take into account individual differences that may influence these processes. For example, individuals who drink more frequently or who drink great amounts of alcohol might be expected to have stronger implicit PAEs because these beliefs are better learned and more highly automatic (Read et al., 2004). In addition, gender differences have been observed in alcohol consumption and expectancies, and past research has demonstrated a stronger association between implicit alcohol expectancies and selfreported drinking in males, particularly with respect to tension reduction PAEs (Kidorf et al., 1995; Read et al., 2004: Thompson et al., 2009). Thus, we included these individual differences in our analyses, controlling for typical drinking and examining gender as a moderator.

\section{Implicitly Measured PAE Processes: Accessibility or Attentional Bias?}

Another issue relevant for understanding implicit PAEs as they relate to drinking involves the nature of implicit PAE processes. Some have argued that implicit PAE processes can be indexed by faster response times to endorse PAE items as this reflects the ease and automaticity with which alcohol beliefs are retrieved from memory (e.g., Palfai et al., 1997; Palfai and Wood, 2001). Others have contended that slower responding to alcohol concepts may be indicative of implicit PAE processes (e.g., Kramer and Goldman, 2003; see also Cox et al., 2006), as slowing may reflect heightened attention to alcohol-relevant cues (including PAE items), which occupy working memory resources and interfere with response times on simultaneous tasks.

Both accessibility and attention bias appear to be important automatic cognitive processes related to drinking, and so it may be that these processes are relevant in different contexts. For example, those PAEs that are more strongly associated with alcohol in memory should be more accessible, and so individuals will respond faster to those items. This may explain why heavier drinkers tend to respond faster to PAE items compared with lighter drinkers (Palfai and Wood, 2001; Read et al., 2004). However, the presentation of salient alcohol cues or an impending drinking situation may activate motivational brain systems in drinkers leading to the desire to drink (see Robinson and Berridge, 1993). In this motivational state, individuals may become preoccupied with motivationally relevant information, including alcohol expectancies (e.g., Kramer and Goldman, 2003; see also Field et al., 2009). This could lead to an attention bias toward these highly salient PAEs, occupying working memory resources and slowing reaction times to endorse these PAEs.

Read and Curtin (2007) have examined how contextual factors may influence the operation of accessibility and attentional bias processes related to PAEs. In this study, participants in a stressful mood condition responded faster to tension reduction PAEs when they were exposed to neutral cues. However, participants in the stressful mood condition who were exposed to alcohol cues had slower reaction times to endorse tension reduction PAEs. The authors argued that activation of implicit PAEs by motivationally relevant (i.e., alcohol) cues may have primed an attentional bias to alcohol information (i.e., PAE items), leading to slower processing and slower response times. In other words, the alcohol cues may have activated these PAEs to the point that they became intrusive and interfered with responding on the task. Thus, context may influence the nature (accessibility vs. attentional bias) of implicit PAE processes.

\section{The Present Study}

Our primary aim was to examine the relationship between implicit PAE processes and subsequent alcohol consumption in the laboratory, and to explore the role of mood in this association. First, we sought to provide a much needed test of the SLT prediction that mood 
congruent implicit PAEs (enhancement, tension reduction) mediate the influence of mood on alcohol consumption. Following previous research that has identified both arousal and valence dimensions of mood effects (see Lang, 1995), we framed our hypotheses on the assumption that both the positive and negative mood conditions would increase arousal, and therefore both would have an influence on latency to endorse PAE items that primarily involve an arousal component. We also anticipated that mood effects would be associated with slower response times to PAEs as all participants were anticipating alcohol consumption and thus were primed by the drinking context to attend to alcohol information (Kramer and Goldman, 2003; Read and Curtin, 2007).

We forwarded the following hypotheses with respect to the proposed mediational model:

1. We predicted that participants in the positive and negative mood conditions would drink more alcohol than those in the neutral mood condition, given that both of these mood states are associated with drinking (e.g., Birch et al., 2008; Cooper et al., 1995).

2. Consistent with SLT, we predicted that the effects of mood on alcohol consumption would be mediated by activation of specific, mood-congruent implicit PAEs. We expected that tension reduction and activity enhancement PAEs would mediate the relationship between mood and in lab drinking, as both of these PAE types involve arousal. We did not expect social lubrication PAEs to act as a mediator as they are not as directly mood-relevant.

3. We also expected an effect of emotional valence; tension reduction RTs would be the strongest mediator of the mood-drinking association for those in the negative mood condition, and enhancement RTs would be the strongest mediator for those in the positive mood condition.

As noted, the role of mood in moderating expectancy effects on drinking has been virtually overlooked in the existing literature. Accordingly, a second objective of the present study was to examine whether mood serves as a moderator of the link between implicit PAEs and subsequent alcohol consumption. We made the following predictions:

1. We expected the association between latency to endorse tension reduction and activity enhancement PAEs and in-lab drinking to be stronger in the context of arousal (positive, negative mood conditions, together) relative to the neutral condition, because both PAE types involve an arousal component. We did not expect mood to moderate the association between social lubrication PAEs and drinking.

2. We expected an effect of emotional valence, such that the association between tension reduction PAEs and drinking would be stronger in the negative mood condition than the positive mood condition, and the opposite would be true for activity enhancement PAEs.

\section{Method}

\section{Participants}

Participants were one hundred forty-six (67 female) college students in the northeastern United States. To be eligible, participants had to be regular drinkers (at least once per week for the past 3 months) between the ages of 21 and 24, without any contraindications to alcohol consumption, and express a liking for beer (which was the beverage used in this study). Eligible students $(N=184)$ completed experimental procedures. Thirty-eight participants were dropped from analyses because they did not endorse at least one item from all three of the PAE scales, and so the final sample consisted of 146 participants. Before the 
session, a random number generator was used to randomly assign participants (within gender) to one of three mood conditions: neutral $(n=43)$, negative $(n=49)$, and positive $(n=54)$. The majority $(n=125,86 \%)$ of participants were Caucasian, $1 \%(n=1)$ were Hispanic, $2 \%(n=3)$ were Asian, $7 \%(n=10)$ were Black, and 3\% $(n=6)$ identified themselves as "Other". One participant did not provide ethnicity data. Over half $(n=81,56 \%)$ were seniors and the average age was $21.45(S D=0.73)$. Participants reported drinking an average of $17.87(S D=12.05)$ drinks per week, on an average of $2.72(S D=1.19)$ weekly drinking occasions. They consumed at least $4 / 5$ (women/men) drinks (heavy episodic drinking) on an average of $7.00(S D=4.27)$ occasions in the last month (see results section for gender differences in drinking habits).

\section{Overview of Procedure}

Study procedures were approved by the university institutional review board. Participants were recruited through advertisements for a study about "college students' beverage taste preferences." Interested individuals completed a phone screen to determine their eligibility. At the beginning of the phone screen, participants were told that they would be asked to taste alcohol as part of the experiment. Eligible participants were scheduled for an appointment in the lab.

All experimental sessions took place in mid-afternoon to control for time of day effects. Sessions were scheduled on weekdays (Monday-Friday). ${ }^{2}$ Upon arrival to the laboratory, female participants were administered hormonal pregnancy tests to confirm non-pregnant status, informed consent was obtained, and breath analysis was administered to ensure that the baseline blood alcohol concentration (BAC) was zero. Participants then completed demographics and baseline mood measures. Following this, the mood induction procedure (MIP; described below) took place and mood measures were re-administered. Next, participants completed a computerized sentence completion task to assess implicit PAEs (ETASK; described below). Mood was re-assessed, and then the Timeline Follow Back interview (Sobell and Sobell, 1992) was administered to assess past 30 day drinking. At this point, approximately 20 minutes had elapsed since the mood induction procedure. So, to prolong the mood effects, a subset of IAPS slides ( $n=8$ for each mood condition) were readministered (see description of MIP below) immediately prior to the alcohol administration.

The ad lib alcohol consumption component was completed next. Each participant was informed that they would engage in a 30 minute beer taste test. The participant was seated alone in a small, neutral room containing a table and chair. The research assistant provided the participant with two pitchers (each containing 24 ounces or 2 standard drinks) of two types of beer. The participant was given a "taste preference" questionnaire and was told that they must taste each type of beer, but that they could drink as much or as little of either or both types as desired. The research assistant checked on the participant and offered to pour more beer into the participant's glass after 5 minutes, and again after 15 minutes. When the participant had finished the taste testing questionnaire, the research assistant entered and reminded the participant that they must remain in this room for the full 30 minutes, and offered to pour more beer. At the end of the 30 minute session, remaining beer was cleared from the table and measured to determine the volume of beer the participant had consumed. Fifteen minutes later, a breath analysis was given and the participant's BAC was recorded. Breath analyses were repeated every 15 minutes until the participants' BAC reached .02, and

\footnotetext{
${ }^{2}$ It is possible that participants, knowing that the study would involve alcohol administration, may have scheduled their session on a day they planned to drink with intentions to use the experiment as a means for "preloading" before going out for the evening. However, because participants were randomly assigned to mood condition, we believe that any potential confounding influence of day of week effects were minimized by equalizing the days on which experiments were run across conditions.
} 
the highest recorded BAC was used as an index of peak BAC achieved in the lab. After participants were debriefed, they were paid $\$ 50$ and released.

Mood induction procedure (MIP)—The MIP was a picture-slide method using the International Affective Picture System (IAPS; Center for the Psychophysiological Study of Emotion and Attention, 1994). This method has been shown to reliably evoke negative, positive, and neutral mood across a number of populations (Davis et al., 1995; Lang, 1995). Moreover, subjective and physiological reactions to the slides map on to the arousal and valence dimensions of human affective responding (see Lang, 1995), with both positive and negative slides eliciting a general arousal response in addition to specific valence responses (pleasant and unpleasant, respectively; Lang et al., 2008).

For the present study, we used the normed mood ratings for the IAPS to select eighty-six slides with the highest average ratings for positive, neutral, and negative mood. Also, we confirmed that slides selected for both the positive and negative mood conditions were high on arousal ratings. The average arousal rating for the subset of IAPS slides that we selected for our negative mood condition $(M=6.06, S D=0.80)$ was higher than the overall average arousal rating $(M=5.04)$ for the entire pool of negatively-valenced IAPS slides, $\mathrm{t}(89)=12.08$, $\mathrm{p}<.001$. The same was true for the mean arousal rating of the slides in the positive mood condition $(M=4.95, \mathrm{~S} D=0.93)$ compared with the pool of positively-valenced IAPS slides $(\mathrm{M}=4.68), \mathrm{t}(89)=2.76, \mathrm{p}=.007$. Moreover, arousal ratings for the positive and negative slides used in this study were significantly higher than the mean arousal rating for the neutral slides that were included in the study $(\mathrm{M}=3.51, \mathrm{SD}=1.04$, $\mathrm{ps}<.001)$.

For the negative and positive conditions, the IAPS slides were paired with non-lyrical classical musical pieces (McKee et al., 2003) to enhance mood effects. Although research assistants were not blind to mood condition, they followed standardized protocols for interacting with participants in order to reduce the potential for experimenter bias. The mood induction procedure lasted for approximately 9 minutes.

Expectancy task (ETASK)—We assessed both implicit and explicit PAEs with a reaction time task developed to measure the accessibility of PAEs from memory, which has also been used as measure of attentional bias to PAE information (Palfai et al., 1997; Read and Curtin, 2007). The ETASK is a computerized sentence completion task, in which participants are asked to respond to a series of PAE items (Kushner et al., 1994). Items used in the present analysis came from the PAE subscales - tension reduction, social lubrication, and activity enhancement - that we have examined in previous work (Read and Curtin, 2007). ${ }^{3}$ Personality trait items were chosen randomly from the Big Five Inventory (John and Srivastava, 1999), and were used to control for individual differences in reaction times. Participants were instructed to respond as quickly and as accurately as possible to PAE and personality statements (e.g., Alcohol helps me ... [1 second delay]... RELAX; Usually I... [1 second delay]... TRUST PEOPLE) by pressing one of two response buttons ("yes" or "no") to indicate whether the stem/target word statement described them. Response times to PAE target words to which participants responded "yes" were averaged across items from each PAE subscale to provide an index of implicit PAEs for each scale (Read and Curtin, 2007). The total number of PAEs endorsed during the task provided an index of explicit PAEs. The ETASK demonstrated adequate reliability in our sample. Reaction times to each expectancy item were adjusted for individual differences in response time (i.e., mean RT to personality items) before calculating internal consistency estimates. Cronbach's alphas for RTs were .84 for tension reduction, .84 for social lubrication, and .78 for activity

\footnotetext{
${ }^{3}$ In order to limit the duration of the ETASK, items from the fourth subscale (performance enhancement; Kushner et al., 1994) were not included in the ETASK (consistent with Read and Curtin, 2007) because they were the least relevant to the mood manipulation.
} 
enhancement. Also, we observed good internal consistency for our index of explicit PAEs in our sample (alpha $=.81)$.

As noted, our use of the term implicit refers to expectancy processes that are measured using indirect methods that do not solely rely upon explicit self-report and are thought to tap into automatic cognitive processes (De Houwer, 2006). For the ETASK, participants were asked to respond to items as quickly as possible, so this reaction time is thought to reflect automatic cognitive processes outside of participants' control (De Houwer, 2006; Fazio and Olson, 2003). Like other measures of implicit PAEs, RTs on the ETASK have been shown to correlate with explicit measures of PAEs as well as self-reported alcohol use (Read et al., 2004; Read and Curtin, 2007).

\section{Measures}

Demographic information-Demographic data gathered in the assessment battery included gender, age, ethnicity, and year in school.

Affect grid-This measure was developed to assess arousal (arousal vs. sedation) and valence (positive vs. negative; Russell et al., 1989) dimensions of affect. Both before and after the mood induction procedure, participants marked an "X" on a 9X9 grid to indicate their current mood within the two-dimensional space. Each dimension was rated on a 9point scale with higher values indicating greater endorsement of that dimension.

Self-reported alcohol use-Past 30 day drinking was assessed with the calendar-based time line follow back method (TLFB; Sobell and Sobell, 1992). This interview uses a visual calendar that includes key dates and maximizes respondent recall (Sobell and Sobell, 1992). Participants reported on the number of standard drinks consumed and the number of hours spent drinking for each day. The TLFB interview took approximately 10 minutes to complete.

Laboratory alcohol consumption-The measured volume of beer consumed during the taste test and the peak BAC measurement recorded following the taste test were the two outcome variables examined in the present study.

\section{Results}

\section{Descriptives and Bivariate Associations}

See Table 1 for descriptive statistics and bivariate correlations. Participants drank an average of $21.81(S D=13.45)$ ounces of beer - nearly 2 standard drinks - during the 30 minute "taste-test". The quantity of beer consumed in lab and peak BAC were positively associated with self-reported drinking (Table 1); heavier drinkers drank more in the lab. The RTs for all three PAE scales were correlated with the personality item RT (see Table 1). Thus, all PAE reaction time analyses included the number of PAEs endorsed and personality RT as covariates. Also, partial correlation analyses controlling for personality item RT showed that the number of PAE items endorsed was negatively correlated with RTs for all 3 PAE scales $(\mathrm{pr}=-.38$ for tension reduction; $\mathrm{pr}=-.32$ for social lubrication; $\mathrm{pr}=-.40$ for activity enhancement; all $p$ s <.001). This shows greater PAE accessibility for those with more explicit PAEs.

We also examined gender differences. Controlling for RT to personality items, there were no gender differences on the RTs to any of the PAE scales (all ps $>.05$ ). However, compared with women, men drank more beer in the lab ( $M=28.84, S D=12.90$ vs. $M=13.51, S D=8.46)$ and had a higher peak BAC ( $M=.045, S D=.024$ vs. $M=.031, S D=.026 ; p s<.01)$. 


\section{Manipulation Check}

We performed analyses of covariance (ANCOVAs) to examine the effect of mood condition on post mood-induction arousal and valence ratings, with baseline ratings as covariates. ${ }^{4} \mathrm{We}$ also included gender as a moderator in the model. After controlling for baseline arousal ratings, there was a significant omnibus effect of mood condition on post-induction arousal, $F(2,138)=5.23, p=.006$. There was no significant effect of gender $(p=.763)$, nor was there a significant interaction between gender and mood condition $(p=.279)$. Contrast tests showed that relative to those in the neutral condition $(M=3.96, S E=0.25)$, significantly greater arousal was reported by participants in both the positive $(M=4.67, S E=0.22 ; p=.033)$ and negative $(M=5.05, S E=0.23 ; p=.002)$ conditions. This confirmed that both positive and negative moods included a component of heightened arousal.

A significant mood condition effect also was observed for post-induction valence ratings, $F(2,138)=93.59, p<.001$. Contrast tests showed that relative to the neutral condition $(M=7.34, S E=0.20)$, participants reported greater pleasant affect in the positive mood condition $(M=7.32, S E=0.19, p=.003)$ and less in the negative mood condition $(M=3.64$, $S E=0.21, p<.001)$. Again, gender and the mood condition $\mathrm{X}$ gender interaction were not significant (ps > .606). Thus, consistent with past research using the IAPS (see Lang, 1995), the mood induction appears to have produced both general arousal (i.e., positive and negative mood conditions vs. neutral) and specific valence effects (i.e., positive vs. negative mood conditions).

\section{Mediation Analysis}

To test the hypothesis that the effects of mood on alcohol consumption would be mediated by latency to endorse specific, mood-congruent PAEs, we followed Baron and Kenny's (1986) steps for testing mediation. According to this procedure, a mediational effect can be present only if two direct effects first are established: (1) the effect of mood condition on alcohol consumption; and (2) the effect of mood condition of latency to endorse PAEs. We included gender and the interaction between mood and gender in our models, to examine gender as a moderator of the mediated pathway.

In order to determine whether mood condition influenced drinking, we conducted analyses of covariance (ANCOVA) with mood condition, gender, and the mood by gender interaction as the independent variables. Typical quantity and frequency of alcohol use were included as covariates. With volume of beer as the dependent variable, we did not observe a significant main effect for mood condition, $F(2,138)=0.66, p=.520$, nor a mood by gender interaction $F(2,138)=1.93, p=.149$. Similarly, with peak in-lab BAC as the dependent variable, there was no significant main effect for mood condition, $F(2,138)=0.35, p=.708$, or $\operatorname{mood}$ by gender interaction $F(2,138)=0.79, p=.456$. There was a significant main effect of gender for both volume, $F(1,138)=43.29, p<.001$, and peak BAC, $F(1,138)=5.24 p=.024$, with means showing that males consumed more beer and had higher BACs than females.

Given that mood condition did not have a direct influence on in-lab alcohol consumption and gender did not moderate the association between mood condition and drinking, the first criterion in the test for mediation was not satisfied. We did not proceed with the mediation analysis as there was no pathway from mood to drinking to be mediated.

\footnotetext{
${ }^{4}$ One participant was excluded from the manipulation check analysis due to missing data on baseline mood measures. Sample sizes for the manipulation check were; neutral $(n=43)$, negative $(n=48)$, positive $(n=54)$. Covariate adjusted means are presented for valence and arousal ratings.
} 


\section{Moderation Analyses}

We next sought to determine whether the effect of PAEs on drinking might be mooddependent (moderated), rather than mood derived (as a mediated pathway would suggest). To do so, we constructed general linear models for each of the two drinking outcomes (peak $\mathrm{BAC}$, volume). Continuous predictors were standardized to facilitate interpretation of the results. Each model included the covariates (PAEs endorsed, RTs to personality items, typical quantity and frequency of drinking) and RTs to three expectancy scales as predictors. To test our specific hypotheses based on the two-dimensional model of human affective response (Russell, 1980; Lang, 1995), two orthogonal mood contrasts were included in each model to probe for arousal (the mean of the positive and negative mood conditions vs. the neutral condition) and valence (negative vs. positive mood conditions) effects. Gender was included in the model to test its role as a moderator. Three-way interactions between each ETASK variable, mood contrast, and gender were tested. Regression diagnostics conducted prior to analyses revealed no multivariate outliers.

Alcohol volume-The overall model accounted for $37 \%$ of the variance in volume of beer consumed, adjusted $R^{2}=.371, F(37,108)=3.31, p<.001$. We observed a significant three-way interaction between gender, the arousal mood contrast, and latency to endorse tension reduction PAEs, $B=-18.43, t(108)=-2.48, p=.015,95 \%$ CI $[-33.18,-3.69]$. The 3-way interactions involving RTs to the other PAE scales were not significant (all $p \mathrm{~s}>.385$ ). To probe the significant 3-way interaction, we examined the 2-way interactions between RT to tension reduction PAEs and mood conditioned on male and female gender. For males, there was a significant 2-way interaction between the arousal mood contrast and latency to endorse tension reduction PAEs, $B=13.91, t(108)=2.70, p=.008,95 \%$ CI $[3.70,24.12]$, but this interaction was not significant for females, $B=-4.52, t(108)=-0.84, p=.401,95 \% \mathrm{CI}$ $[-15.15,6.10]$. We probed the 2-way interaction for men and found a significant simple effect of latency to endorse tension reduction PAEs in the context of arousal, $B=7.42$, $t(108)=3.58, p=.001,95 \%$ CI [3.31, 11.53], but not neutral mood, $B=-6.49, t(108)=-1.38$, $p=.171,95 \% \mathrm{CI}[-15.83,2.85]$. So, latency to endorse tension reduction PAEs was a stronger predictor of volume of alcohol consumed in the context of arousal (i.e., positive and negative mood conditions) compared with neutral mood, and this effect applied only to men (See Figure 1).

Peak BAC—The overall model accounted for $8.0 \%$ of the variance in BAC, adjusted $R^{2}=$. $080, F(37,108)=1.34, p=123.5$ The pattern of findings for peak BAC mirrored that for volume. There was a significant three-way interaction between gender, the arousal mood contrast, and latency to endorse tension reduction PAEs, $B=-.035, t(108)=-2.00, p=.048$, 95\% CI [-.069, -.001], but none of the other 3-way interactions were significant (all $p \mathrm{~s}>$. 420 ). Inspection of the conditional 2 -way interactions revealed a significant interaction between the arousal mood contrast and RTs for tension reduction PAEs for men, $B=.028$, $t(108)=2.34, p=.021,95 \%$ CI $[.004, .052]$ but not women, $B=-.007, t(108)=-0.52, p=.602$, $95 \%$ CI $[-.031, .018]$. Again, when conditioned on male gender, there was a significant simple effect of latency to endorse tension reduction PAEs in the context of arousal, $B=.013$, $t(108)=2.60, p=.011,95 \%$ CI $[.003, .022]$, but not neutral mood, $B=-.016, t(108)=-1.41$, $p=.161,95 \%$ CI $[-.037, .006]$ (Figure 2).

\footnotetext{
${ }^{5}$ The overall model predicting in-lab BAC was not significant, which may be partially a result of the large number of nonsignificant interaction terms in the model. However, because we had specific, a priori hypotheses about the unique influence of PAEs on drinking in particular mood contexts, we probed significant interactions and interpreted significant regression coefficients despite the nonsignificance of the overall model (Bedeian and Mossholder, 1994).
} 


\section{Discussion}

As interest in implicit alcohol expectancy processes grows, evidence that these processes predict actual proximate alcohol consumption is essential. Remarkably, with only a few exceptions (e.g., Ostafin et. al., 2008; Payne et al., 2008; Roehrich and Goldman, 1995; Stein et al., 2000), little research has attempted to acquire this evidence. The present study provides a theory-driven examination of implicitly measured PAE processes as determinants of immediate alcohol consumption. Rather than confirming the mediational hypothesis derived from a social learning theory (SLT) perspective, our data supported an alternative model, suggesting that both mood and gender moderate the link between implicit PAEs and immediate alcohol consumption. The findings of this study are consistent with expectancy theory, which posits that the influence of PAEs on drinking emerge only in relevant contexts (Goldman et al., 1999; Stein et al., 2000).

\section{Mood, Gender, PAE Processes, and Alcohol Consumption}

We found that response times to tension reduction PAEs more strongly predicted drinking and peak in-lab BAC in the context of arousal relative to neutral affect. Moreover, this effect was observed only for men, a finding that is consistent with past studies (Kidorf et al., 1995; Read et al., 2004; Thompson et al., 2009). This may reflect the tendency for men to have more ingrained, habitual patterns of using alcohol for tension reduction reasons (Cooper et al., 1992). Over time, repeatedly associating alcohol use with tension reduction may lead to greater automaticity of tension reduction PAEs, along with a stronger relationship between these implicit PAEs and drinking.

Also, because tension reduction PAEs generally pertain to relaxation and calming effects (e.g., "drinking helps me to relax"), it stands to reason that they may relate to drinking behavior in the context of general arousal regardless of valence (positive or negative). Indeed, many of the items did not include specific emotional valence content (e.g., "drinking helps me to relax," "drinking helps me deal with boredom"). Though we predicted that affective valence also would play a role, the association did not differ across positive and negative mood conditions for either gender.

The fact that we did not find support for our prediction that mood would influence the association between activity enhancement PAEs and drinking may again reflect the importance of context. Because activity enhancement PAEs reference excitement and stimulation, they may have been less relevant for influencing drinking as there was not much opportunity for excitement or stimulation in the lab context. Instead, it seems that tension reduction PAEs - which deal with relaxation promotion, boredom reduction, etc. - may have been more relevant.

The magnitudes of the effects support the practical significance of these findings. For example, in the context of arousal, a one standard deviation increase in latency to endorse tension reduction PAEs predicted an increase of $7.42 \mathrm{oz}$ of beer consumed for men - more than half of a standard drink. For peak BAC, a one standard deviation increase in tension reduction PAE response latency predicted a BAC increase of .013 for men. Given that the mean peak BAC in this sample was .04, it is notable that these reaction times meaningfully predicted BACs even when drinking was being observed non-naturalistically (in a laboratory mid-afternoon).

\section{Accessibility vs. Attentional Bias}

Our data speak to the question of whether implicitly measured PAE processes that influence immediate drinking reflect greater accessibility of alcohol beliefs from memory or biased attention to alcohol-relevant information. Consistent with past research (e.g., Palfai and 
Wood 2001; Read et al., 2004; Read and Curtin, 2007), we found that individuals with stronger explicit PAEs were quicker to access these PAEs from memory. However, our data also show that PAE accessibility may not be the process that is most relevant to proximal drinking behavior. In this study, it was slower reaction time to PAEs that was associated with greater alcohol consumption and peak BAC in the relevant mood context. This finding is consistent with those of Read and Curtin (2007), who found that exposure to alcohol cues (vs. neutral cues) lead to slower PAE reaction time (see also Kramer and Goldman, 2003). In the present study, participants were "cued" by the anticipation of an imminent drinking situation, and this cue may have primed the motivation to drink (Robinson and Berridge, 1993). As noted before, slower reaction times to PAE items may occur when PAEs match a current motivational state; rather than facilitating endorsement, they automatically capture attention to the degree that they deplete resources needed to make a response on the reaction time task.

Given the dearth of research examining PAE processes relative to actual drinking behavior, our finding that RTs to PAE items predicted the amount of alcohol consumed in the lab represents a significant contribution. That males who exhibited slower RTs to mood-relevant PAE items also consumed more alcohol and achieved higher BACs is evidence that implicitly measured PAE processes have an influence over in-the-moment drinking. In a drinking situation, drinking depends not only on stable, declarative beliefs about alcohol's positive effects, but also on mood-relevant automatic cognitive processes, at least for men.

\section{Social Learning Theory and the Mediation Hypothesis}

With this study we have provided a much needed experimental test of a dominant SLTbased model describing a mediated pathway from mood to mood-relevant expectancy processes, and eventually, to drinking. Based on this model, we expected to find that latency to endorse specific PAEs would mediate the relation between mood condition and alcohol consumption. However, this hypothesis was not supported as mood condition did not influence in-lab drinking.

The lack of direct effects of mood condition on in-lab alcohol consumption is inconsistent with at least some prior work (e.g. Noel and Lisman, 1980). It may be that the type of affect induced by looking at pictures and listening to classical music was not analogous to the type of mood states that might influence real-world drinking patterns for college students (e.g., stress over failing an exam, excitement created by a party, etc.). It also is possible that the mood effects did not continue into the drinking paradigm, as there was a time lag between the initial mood induction procedure and the alcohol administration. Although we did readminister a subset of the IAPS slides just prior to the drinking paradigm with the intention of prolonging the mood effects, we do not have a measure of mood immediately preceding drinking, which limits our ability to determine whether the groups differed on mood immediately before drinking commenced. Future studies could attempt to address this limitation by positioning the ad lib consumption component of the study closer in time to the mood induction procedure.

\section{Future Directions}

The present study points to several directions for continued inquiry. First, there are aspects of the ETASK which limit the interpretation of these indices as pure measures of explicit and implicit processes. For example, the correlation between endorsement frequency and reaction time might be influenced by factors particular to the task, and so interpreting these as measures of explicit vs. implicit PAEs may not be straightforward. Furthermore, rather than instructing participants to ignore the semantic content of items as is done in other tasks measuring attention bias (e.g., the Stroop task; Cox et al., 2006), the ETASK requires 
participants to consciously processes the semantic content of the items. Thus, slower reaction time in this task may be a function of explicit attention allocation and deliberative processing of motivationally-relevant information. Future work should incorporate additional measures to help disentangle explicit and implicit expectancy processes.

Another limitation was the homogeneity of the sample, which consisted of fairly heavy drinking college students. Future research should examine these processes in less alcoholinvolved individuals and clinical populations. Future studies also should incorporate drinking paradigms with improved ecological validity (i.e., examinations in contexts more typical of real world drinking situations) to further increase the generalizability of the findings. In our study we sought to minimize the potential influence of experimenter bias by standardizing the protocol across conditions. Still, our research assistants were not blind to mood condition and thus we cannot rule out the possibility of experimenter bias.

In this study the positive and negative mood conditions were associated with higher arousal compared with the neutral mood condition. Thus, we interpreted the moderating effect of mood condition as a function of differences in arousal. Yet, a more direct test of the moderating role of arousal in the association between implicit PAEs and drinking would involve direct assessment of individual differences on arousal, which would require the use of a more comprehensive and reliable measure of mood than was used in the present study. Thus, future studies are needed to assess the role of individual differences in mood in the link between PAEs and alcohol consumption.

\section{Implications and Conclusions}

Our findings show that drinking behavior may be at least partially influenced by automatic cognitive processes. We found that mood moderates the association between implicit PAEs and alcohol consumption. These findings are consistent with expectancy theory, which posits that the influence of PAEs on drinking will emerge only in a relevant context. Thus, expectancy-based interventions should target implicit PAEs in addition to explicit ones. This is especially important because it has been argued that implicit alcohol-related cognitions have a powerful, automatic influence over drinking behavior that may thwart conscious attempts to regulate alcohol consumption, especially when self-control resources are depleted (Ostafin et al., 2008; Wiers \& Stacy, 2006). The development of interventions that target implicit cognition is underway (Wiers and Stacy, 2006). Also, because the influence of implicit PAEs on immediate drinking behavior appears to be most pronounced in particular mood contexts, interventions might focus on helping individuals recognize the mood conditions under which their positive beliefs about alcohol are most likely to guide their immediate drinking behavior. In particular, teaching individuals to exercise conscious control over drinking in risky mood states may help reduce the impact that implicit PAEs have on alcohol consumption.

\section{Acknowledgments}

This research was supported in part by a grant from the National Institute on Alcohol Abuse and Alcoholism (AAR21014052) to Dr. Jennifer P. Read. We thank Dr. Stephen T. Tiffany for his helpful feedback on an earlier version of this manuscript; and Amanda Ayler, Rachel Bachrach, Joseph Bardeen, Lisa Braun, Rebecca Chamberlain, Morgan Gottfried, Melissa Griffin, Urmo Jaanimagi, Carol Malik, Stephanie Torkelson, and Leah Vermont for assistance with data collection and feedback on the manuscript.

\section{References}

Baron RM, Kenny DA. The moderator-mediator variable distinction in social psychological research. J Pers Soc Psychol. 1986; 51:1173-1182. [PubMed: 3806354] 
Bedeian AG, Mossholder KW. Simple question, not so simple answer: Interpreting interaction terms in moderated multiple regression. J Manage. 1994; 20:159-165.

Birch CD, Stewart SH, Wiers RW, Klein RM, MacLean AD, Berish MJ. The mood-induced activation of implicit alcohol cognition in enhancement and coping motivated drinkers. Addict Behav. 2008; 33:565-581. [PubMed: 18155854]

Birch CD, Stewart SH, Wall A-M, McKee SA, Eisnor SJ, Theakson JA. Mood-induced increases in alcohol expectancy strength in internally motivated drinkers. Addict Behav. 2004; 18:231-238.

Bolles RC. Reinforcement, expectancy, and learning. Psychol Rev. 1972; 79:394-409.

Center for the Psychophysiological Study of Emotion and Attention. The International Affective Picture System. Center for Research in Psychophysiology. University of Florida; Gainsville, FL: 1994.

Cooper ML, Frone MR, Russell M, Mudar P. Drinking to regulate positive and negative emotions: A motivational model of alcohol use. J Pers Soc Psychol. 1995; 69:990-1005. [PubMed: 7473043]

Cooper ML, Russell M, Skinner JB, Frone MR, Mudar P. Stress and alcohol use: Moderating effects of gender, coping, and alcohol expectancies. J J Abnorm Psychol. 1992; 101:139-152.

Cox WM, Fadardi JS, Pothos EM. The addiction-stroop test: theoretical considerations and procedural recommendations. Psychol Bull. 2006; 132:443-476. [PubMed: 16719569]

Davis WJ, Rahman MA, Smith LJ, Burns A, Senecal L, McArthur D, Halpern JA, Perlmutter A, Sickels W, Wagner W. Properties of human affect induced by static color slides (IAPS): dimensional, categorical, and electromyographic analysis. Biol Psychol. 1995; 41:229-253. [PubMed: 8608202]

De Houwer, J. What are implicit measures and why are we using them?. In: Wiers, RW.; Stacy, AW., editors. Handbook of Implicit Cognition and Addiction. Sage Publications; Thousand Oaks, CA: 2006. p. 11-28.

Fazio RH, Olson MA. Implicit measures in social cognition research: their meaning and uses. Annu Rev Psychol. 2003; 54:297-327. [PubMed: 12172003]

Field M, Muafo MR, Franken IHA. A meta-analytic investigation of the relationship between attentional bias and subjective craving in substance abuse. Psychol Bull. 2009; 135:589-607. [PubMed: 19586163]

Friedman RS, McCarthy DM, Pedersen SL, Hicks JA. Alcohol expectancy priming and drinking behavior: the role of compatibility between prime and expectancy content. Psychol Addict Behav. 2009; 23:329-333. [PubMed: 19586149]

Goldman, MS.; Del Boca, FK.; Darkes, J. Alcohol expectancy theory: the application of cognitive neuroscience. In: Leonard, KE.; Blane, HT., editors. Psychological Theories of Drinking and Alcoholism. Guilford Press; New York: 1999. p. 203-246.

Goldstein AL, Wall A-M, McKee SA, Hinson RE. Accessibility of alcohol expectancies from memory: impact of mood and motives in college student drinkers. J Stud Alcohol. 2004; 65:95104. [PubMed: 15000508]

Grant V, Stewart SH. Impact of experimentally induced positive and anxious mood on alcohol expectancy strength in internally motivated drinkers. Cogn Behav Ther. 2007; 36:102-111. [PubMed: 17530496]

Greeley, J.; Oei, T. Alcohol and tension reduction, in Psychological Theories of Drinking and Alcoholism. Leonard, KE.; Blane, HT., editors. Guilford Press; New York: 1999. p. 14-53.

Houben K, Wiers RW. Implicitly positive about alcohol? Implicit positive associations predict drinking behavior. Addict Behav. 2008; 33:979-986. [PubMed: 18434034]

Hufford MR. An examination of mood effects on positive alcohol expectancies among undergraduate drinkers. Cognition Emotion. 2001; 15:93-613.

John, OP.; Srivastava, S. The big five trait taxonomy: history, measurement, and theoretical perspectives. In: Pervin, LA.; John, OP., editors. Handbook of Personality: Theory and Research. Guilford Press; New York: 1999. p. 102-138.

Kidorf M, Sherman MF, Johnson JG, Bigelow GE. Alcohol expectancies and changes in beer consumption of first year college students. Addict Behav. 1995; 20:225-231. [PubMed: 7484316] 
Kramer DA, Goldman MS. Using a modified stroop task to implicitly discern the cognitive organization of alcohol expectancies. J Abnorm Psychol. 2003; 112:171-175. [PubMed: 12653426]

Krank, MD.; Wall, A-M. Context and retrieval effects on implicit cognition for substance use. In: Wiers, RW.; Stacy, AW., editors. Handbook of Implicit Cognition and Addiction. Sage Publications; Thousand Oaks, CA: 2006. p. 281-292.

Kushner MG, Sher KJ, Wood MD, Wood PK. Anxiety and drinking behavior: moderating effects of tension-reduction alcohol outcome expectancies. Alcohol Clin Exp Res. 1994; 18:852-860. [PubMed: 7978095]

Lang PJ. The emotion probe: studies of motivation and attention. Am Psychol. 1995; 50:372-385. [PubMed: 7762889]

Lang, PJ.; Bradley, MM.; Cuthbert, BN. Technical report 8-A. University of Florida; Gainsville, FL: 2008. International affective picture system (IAPS): Affective ratings of pictures and instruction manual.

Maisto, SA.; Carey, KB.; Bradizza, CM. Social learning theory, in Psychological Theories of Drinking and Alcoholism. Leonard, KE.; Blane, HT., editors. Guilford Press; New York: 1999. p. 106-163.

Mann LM, Chassin L, Sher KJ. Alcohol expectancies and the risk for alcoholism. J Consult Clin Psych. 1987; 55:411-417.

McCarthy DM, Thompsen DM. Implicit and explicit measures of alcohol and smoking cognitions. Psychol Addict Behav. 2006; 20:436-444. [PubMed: 17176178]

McKee SA, Wall A-M, Hinson RE, Goldstein A, Bissonnette M. Effects of implicit mood prime on the accessibility of smoking expectancies in college women. Psychol Addict Behav. 2003; 17:219225. [PubMed: 14498816]

Noel NE, Lisman SA. Alcohol consumption by college women following exposure to unsolvable problems: learned helplessness or stress-induced drinking? Behav Res Ther. 1980; 18:429-440. [PubMed: 7436990]

Ostafin BD, Marlatt GA, Greenwald AG. Drinking without thinking: an implicit measure of alcohol motivation predicts failure to control alcohol use. Behav Res Ther. 2008; 46:1210-1219. [PubMed: 18823876]

Palfai TP, Monti PM, Colby SM, Rohsenow DJ. Effects of suppressing the urge to drink on the accessibility of alcohol outcome expectancies. Behav Res Ther. 1997; 35:59-65. [PubMed: 9009044]

Palfai TP, Monti PM, Ostafin B, Hutchison K. Effects of nicotine deprivation on alcohol-related information processing and drinking behavior. J Abnorm Psychol. 2000; 109:96-105. [PubMed: 10740940]

Palfai T, Wood MD. Positive alcohol expectancies and drinking behavior: the influence of expectancy strength and memory accessibility. Psychol Addict Behav. 2001; 15:60-67. [PubMed: 11255940]

Payne BK, Govorun O, Arbuckle NL. Automatic attitudes and alcohol: does implicit liking predict drinking? Cognition Emotion. 2008; 22:238-271.

Pohorecky L. Biphasic action of alcohol. Biobehav Rev. 1977; 1:231-240.

Rather BC, Goldman MS, Roehrich L, Brannick M. Empirical modeling of an alcohol expectancy memory network using multidimensional scaling. J Abnorm Psychol. 1992; 101:174-183. [PubMed: 1537963]

Read JP, Curtin JJ. Contextual influences on alcohol expectancy processes. J Stud Alcohol Drugs. 2007; 68:759-770. [PubMed: 17690810]

Read JP, Lau-Barraco C, Dunn ME, Borsari B. Projected alcohol dose influences on the activation of alcohol expectancies in college drinkers. Alcohol Clin Exp Res. 2009; 33:1265-1277. [PubMed: 19389186]

Read JP, Wood MD, Lejuez CW, Palfai TP, Slack M. Gender, alcohol consumption, and differing alcohol expectancy dimensions in college drinkers. Exper Clin Psychopharm. 2004; 12:298-308.

Robinson TE, Berridge KC. The neural basis of drug craving: An incentive-sensitization theory of addiction. Brain Res Rev. 1993; 18:247-291. [PubMed: 8401595]

Roehrich L, Goldman MS. Implicit priming of alcohol expectancy memory processes and subsequent drinking behavior. Exper Clin Psychopharm. 1995; 3:402-410. 
Russell JA. A circumplex model of affect. J Pers Soc Psychol. 1980; 39:1161-1178.

Russell JA, Weiss A, Mendelsohn GA. Affect grid: a single-item measure of pleasure and arousal. J Pers Soc Psychol. 1989; 57:493-502.

Sher KJ, Wood MD, Wood PK, Raskin G. Alcohol outcome expectancies and alcohol use: a latent variable cross-lagged panel study. J Abnorm Psychol. 1996; 105:561-574. [PubMed: 8952189]

Simons JS, Gaher RM, Oliver MNI, Palmer JA, Bush MA. An experience sampling study of association between affect and alcohol use and problems among college students. J Stud Alcohol. 2005; 66:285-304.

Sobell, LC.; Sobell, M. Timeline follow-back: a technique for assessing self-reported alcohol consumption. In: Litten, RZ.; Allen, JP., editors. Measuring Alcohol Consumption: Psychosocial and Biochemical Methods. Humana Press; Totowa, NJ: 1992. p. 41-72.

Stein KD, Goldman MS, Del Boca FK. The influence of alcohol expectancy priming and mood manipulation on subsequent alcohol consumption. J Abnorm Psychol. 2000; 109:106-115. [PubMed: 10740941]

Stewart SH, Hall E, Wilkie H, Birch C. Affective priming of alcohol schema in coping and enhancement motivated drinkers. Cogn Behav Ther. 2002; 31:68-80.

Tiffany ST. A cognitive model of drug urges and drug-use behavior: the role of automatic and nonautomatic processes. Psychol Rev. 1990; 97:147-168. [PubMed: 2186423]

Thompson MP, Spitler H, McCoy TP, Marra L, Sutfin EL, Rhodes SD, Brown C. The moderating role of gender in the prospective associations between expectancies and alcohol-related negative consequences among college students. Subst Use Misue. 2009; 44:934-942.

Thrush C, Wiers RW. Explicit and implicit alcohol-related cognitions and the prediction of future drinking in adolescents. Addict Behav. 2007; 32:1367-1383. [PubMed: 17125932]

Wiers, RW.; Houben, K.; Smulders, FTY.; Conrod, PJ.; Jones, BT. To drink or not to drink: the role of automatic and controlled cognitive processes in the etiology of alcohol-related problems. In: Wiers, RW.; Stacy, AW., editors. Handbook of Implicit Cognition and Addiction. Sage Publications; Thousand Oaks, CA: 2006. p. 91-104.

Wiers RW, Stacy AW. Implicit cognition and addiction. Curr Dir Psychol Sci. 2006; 15:292-296.

Wiers RW, Stacy AW, Ames SL, Noll JA, Sayette MA, Zack M, Krank M. Implicit and explicit alcohol-related cognition. Alcohol Clin Exp Res. 2002; 26:129-137. [PubMed: 11821663] 


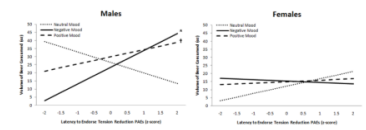

Figure 1.

Association between reaction time (RT) to tension reduction expectancies (z-score) and volume of beer consumed (oz) in the lab by gender and mood condition. A significant interaction between mood condition and RT to tension reduction expectancies was found for males but not females. *Simple slope is significant at .05 level. ${ }^{\dagger}$ Simple slope is marginally significant at .10 level. 


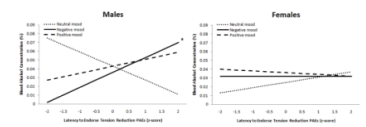

Figure 2.

Association between reaction time (RT) to tension reduction expectancies and peak blood alcohol concentration (BAC) in the lab by gender and mood condition. A significant interaction between mood condition and RT to tension reduction expectancies was found for males but not females. *Simple slope is significant at .05 level. 


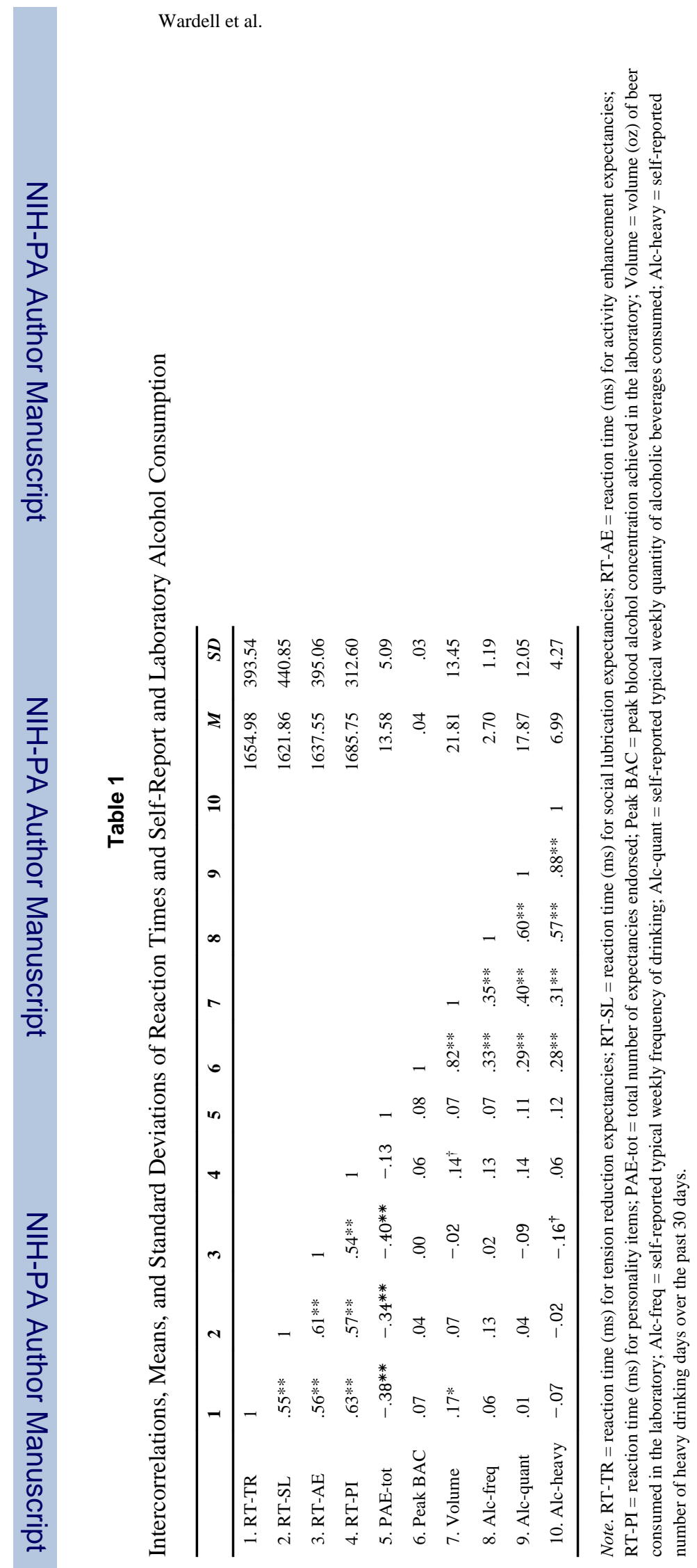

Alcohol Clin Exp Res. Author manuscript; available in PMC 2013 January 1. 